Article

\title{
On the Solvability of Nonlinear Third-Order Two-Point Boundary Value Problems
}

\author{
Ravi P. Agarwal ${ }^{1, *}$, Petio S. Kelevedjiev ${ }^{2}$ and Todor Z. Todorov ${ }^{2}$
}

1 Department of Mathematics, Texas A and M University-Kingsville, Kingsville, TX 78363-8202, USA

2 Department of Mathematics, Technical University of Sliven, 8800 Sliven, Bulgaria; pskeleved@abv.bg (P.S.K.); tjtodorov@abv.bg (T.Z.T.)

* Correspondence: agarwal@tamuk.edu

Received: 21 April 2020; Accepted: 23 May 2020; Published: 31 May 2020

check for updates

Abstract: Under barrier strips type assumptions we study the existence of $C^{3}[0,1]$ 一 solutions to various two-point boundary value problems for the equation $x^{\prime \prime \prime}=f\left(t, x, x^{\prime}, x^{\prime \prime}\right)$. We give also some results guaranteeing positive or non-negative, monotone, convex or concave solutions.

Keywords: third-order differential equation; boundary value problem; existence; sign conditions

MSC: 34B15; 34B18

\section{Introduction}

In this paper, we study the solvability of boundary value problems (BVPs) for the differential equation

$$
x^{\prime \prime \prime}=f\left(t, x, x^{\prime}, x^{\prime \prime}\right), t \in(0,1),
$$

with some of the boundary conditions

$$
\begin{aligned}
& x(0)=A, x^{\prime}(1)=B, x^{\prime \prime}(1)=C, \\
& x(0)=A, x^{\prime}(0)=B, x^{\prime \prime}(1)=C, \\
& x(0)=A, x(1)=B, x^{\prime \prime}(1)=C, \\
& x(0)=A, x^{\prime}(0)=B, x^{\prime}(1)=C, \\
& x(1)=A, x^{\prime}(0)=B, x^{\prime}(1)=C,
\end{aligned}
$$

where $f:[0,1] \times D_{x} \times D_{p} \times D_{q} \rightarrow \mathbb{R}, D_{x}, D_{p}, D_{q} \subseteq \mathbb{R}$, and $A, B, C \in \mathbb{R}$.

The solvability of BVPs for third-order differential equations has been investigated by many authors. Here, we will cite papers devoted to two-point BVPs which are mostly with some of the above boundary conditions; in each of these works $A, B, C=0$. Such problems for equations of the form

$$
x^{\prime \prime \prime}=f(t, x), t \in(0,1),
$$

have been studied by H. Li et al. [1], S. Li [2] (the problem may be singular at $t=0$ and/or $t=1$ ), Z. Liu et al. [3,4], X. Lin and Z. Zhao [5], S. Smirnov [6], Q. Yao and Y. Feng [7]. Moreover, the boundary conditions in References [2,3] are (3), in Reference [4] they are (4), in References [1,5,7] they are (5), and in Reference [6] are

$$
x(0)=x(1)=0, x^{\prime}(0)=C .
$$


Y. Feng [8] and Y. Feng and S. Liu [9] have considered the equation

$$
x^{\prime \prime \prime}=f\left(t, x, x^{\prime}\right), t \in(0,1),
$$

with (6) and (5), respectively. Y. Feng [10] and R. Ma and Y. Lu [11] have studied the equations

$$
f\left(t, x, x^{\prime}, x^{\prime \prime \prime}\right)=0 \text { and } x^{\prime \prime \prime}+M x^{\prime \prime}+f(t, x)=0, t \in(0,1) .
$$

with (5). BVPs for the equation

$$
x^{\prime \prime \prime}=f\left(t, x, x^{\prime}, x^{\prime \prime}\right), t \in(0,1),
$$

have been investigated by A. Granas et al. [12], B. Hopkins and N. Kosmatov [13], Y. Li and Y. Li [14]; the boundary conditions in [12] are (5), these in Reference [13] are (2) and (3), and in Reference [14] - (2).

Results guaranteeing positive or non-negative solutions can be found in References [2-4,7-11,13,14], and results that guarantee negative or non-positive ones in References $[7,9,10]$. The existence of monotone solutions has been studied in References [3,7,9].

As a rule, the main nonlinearity is defined and continuous on a set such that each dependent variable changes in a left- and/or a right-unbounded set; in Reference [13] it is a Carathéodory function on an unbounded set. Besides, the main nonlinearity is monotone with respect to some of the variables in References [1,5], does not change its sign in References [2-4,14] and satisfies Nagumo type growth conditions in Reference [14]. Maximum principles have been used in References [8,10], Green's functions in References [1,2,4,5], and upper and lower solutions in References [1,7-11].

Here, we use a different tool-barrier strips which allow the right side of the equation to be defined and continuous on a bounded subset of its domain and to change its sign.

To prove our existence results we apply a basic existence theorem whose formulation requires the introduction of the BVP

$$
\begin{aligned}
x^{\prime \prime \prime}+a(t) x^{\prime \prime}+b(t) x^{\prime}+c(t) x & =f\left(t, x, x^{\prime}, x^{\prime \prime}\right), t \in(0,1), \\
V_{i}(x)=r_{i}, i=1,2,3(i= & \overline{1,3} \text { for short }),
\end{aligned}
$$

where $a, b, c \in C([0,1], \mathbb{R}), f:[0,1] \times D_{x} \times D_{p} \times D_{q} \rightarrow \mathbb{R}$,

$$
V_{i}(x)=\sum_{j=0}^{2}\left[a_{i j} x^{(j)}(0)+b_{i j} x^{(j)}(1)\right], i=\overline{1,3},
$$

with constants $a_{i j}$ and $b_{i j}$ such that $\sum_{j=0}^{2}\left(a_{i j}^{2}+b_{i j}^{2}\right)>0, i=\overline{1,3}$, and $r_{i} \in \mathbb{R}, i=\overline{1,3}$. Next, consider the family of BVPs for

$$
x^{\prime \prime \prime}+a(t) x^{\prime \prime}+b(t) x^{\prime}+c(t) x=g\left(t, x, x^{\prime}, x^{\prime \prime}, \lambda\right), t \in(0,1), \lambda \in[0,1]
$$

with boundary conditions (8), where $g$ is a scalar function defined $[0,1] \times D_{x} \times D_{p} \times D_{q} \times[0,1]$, and $a, b, c$ are as above. Finally, $B C$ denotes the set of functions satisfying boundary conditions (8), and $B C_{0}$ denotes the set of functions satisfying the homogeneous boundary conditions $V_{i}(x)=0, i=\overline{1,3}$. Besides, let $C_{B C}^{3}[0,1]=C^{3}[0,1] \cap B C$ and $C_{B C_{0}}^{3}[0,1]=C^{3}[0,1] \cap B C_{0}$.

The proofs of our existence results are based on the following theorem. It is a variant of Reference [12] (Chapter I, Theorem 5.1 and Chapter V, Theorem 1.2). Its proof can be found in Reference [15]; see also the similar result in Reference [16] (Theorem 4).

Lemma 1. Suppose:

(i) Problem (7) $)_{0}$, (8) has a unique solution $x_{0} \in C^{3}[0,1]$.

(ii) Problems (7), (8) and (7) 1 , (8) are equivalent. 
(iii) The map $\mathbf{L}_{h}: C_{B C_{0}}^{3}[0,1] \rightarrow C[0,1]$ is one-to-one: here,

$$
\mathbf{L}_{h} x=x^{\prime \prime \prime}+a(t) x^{\prime \prime}+b(t) x^{\prime}+c(t) x .
$$

(iv) Each solution $x \in C^{3}[0,1]$ to family $(7)_{\lambda}$, (8) satisfies the bounds

$$
m_{i} \leq x^{(i)} \leq M_{i} \text { for } t \in[0,1], i=\overline{0,3},
$$

where the constants $-\infty<m_{i}, M_{i}<\infty, i=\overline{0,3}$, are independent of $\lambda$ and $x$.

(v) There is a sufficiently small $\sigma>0$ such that

$$
\left[m_{0}-\sigma, M_{0}+\sigma\right] \subseteq D_{x},\left[m_{1}-\sigma, M_{1}+\sigma\right] \subseteq D_{p},\left[m_{2}-\sigma, M_{2}+\sigma\right] \subseteq D_{q},
$$

and $g(t, x, p, q, \lambda)$ is continuous for $(t, x, p, q, \lambda) \in[0,1] \times J \times[0,1]$ where $J=\left[m_{0}-\sigma, M_{0}+\sigma\right] \times\left[m_{1}-\right.$ $\left.\sigma, M_{1}+\sigma\right] \times\left[m_{2}-\sigma, M_{2}+\sigma\right] ; m_{i}, M_{i}, i=\overline{0,3}$, are as in (iv).

Then boundary value problem (7), (8) has at least one solution in $C^{3}[0,1]$.

For us, the equation from $(7)_{\lambda}$ has the form

$$
x^{\prime \prime \prime}=\lambda f\left(t, x, x^{\prime}, x^{\prime \prime}\right) .
$$

Preparing the application of Lemma 1, we impose conditions which ensure the a priori bounds from (iv) for the eventual $C^{3}[0,1]$ - solutions of the families of BVPs for $(7)_{\lambda}, \lambda \in[0,1]$, with one of the boundary conditions $(k), k=\overline{2,6}$.

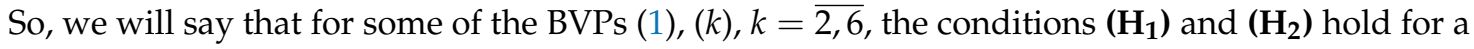
$K \in \mathbb{R}$ (it will be specified later for each problem) if:

$\left(\mathbf{H}_{1}\right)$ There are constants $F_{i}^{\prime}, L_{i}^{\prime}, i=1,2$, such that

$$
\begin{gathered}
F_{2}^{\prime}<F_{1}^{\prime} \leq K \leq L_{1}^{\prime}<L_{2}^{\prime},\left[F_{2}^{\prime}, L_{2}^{\prime}\right] \subseteq D_{q}, \\
f(t, x, p, q) \geq 0 \text { for }(t, x, p, q) \in[0,1] \times D_{x} \times D_{p} \times\left[L_{1}^{\prime}, L_{2}^{\prime}\right], \\
f(t, x, p, q) \leq 0 \text { for }(t, x, p, q) \in[0,1] \times D_{x} \times D_{p} \times\left[F_{2}^{\prime}, F_{1}^{\prime}\right] .
\end{gathered}
$$

(H) There are constants $F_{i}, L_{i}, i=1,2$, such that

$$
\begin{gathered}
F_{2}<F_{1} \leq K \leq L_{1}<L_{2},\left[F_{2}, L_{2}\right] \subseteq D_{q}, \\
f(t, x, p, q) \leq 0 \text { for }(t, x, p, q) \in[0,1] \times D_{x} \times D_{p} \times\left[L_{1}, L_{2}\right], \\
f(t, x, p, q) \geq 0 \text { for }(t, x, p, q) \in[0,1] \times D_{x} \times D_{p} \times\left[F_{2}, F_{1}\right] .
\end{gathered}
$$

Besides, we will say that for some of the BVPs (1), $(k), k=\overline{2,6}$, the condition $\left(\mathbf{H}_{3}\right)$ holds for constants $m_{i} \leq M_{i}, i=\overline{0,2}$, (they also will be specified later for each problem) if:

$\left.\mathbf{( H}_{3}\right)\left[m_{0}-\sigma, M_{0}+\sigma\right] \subseteq D_{x},\left[m_{1}-\sigma, M_{1}+\sigma\right] \subseteq D_{p},\left[m_{2}-\sigma, M_{2}+\sigma\right] \subseteq D_{q}$ and $f(t, x, p, q)$ is continuous on the set $[0,1] \times J$, where $J$ is as in $(v)$ of Lemma 1 , and $\sigma>0$ is sufficiently small.

In fact, the present paper supplements P. Kelevedjiev and T. Todorov [15] where only conditions $\left(\mathbf{H}_{2}\right)$ and $\left(\mathbf{H}_{3}\right)$ have been used for studying the solvability of various BVPs for (1) with other boundary conditions. Here, $\left(\mathbf{H}_{\mathbf{1}}\right)$ is also needed. Now, only $\left(\mathbf{H}_{\mathbf{1}}\right)$ guarantees the a priori bounds for $x^{\prime \prime}(t), x^{\prime}(t)$ and $x(t)$, in this order, for each eventual solution $x \in C^{3}[0,1]$ to the families $(1)_{\lambda},(k), k=\overline{2,4}$, and $\left(\mathbf{H}_{1}\right)$ and $\left(\mathbf{H}_{\mathbf{2}}\right)$ together guarantee these bounds for the families $(1)_{\lambda},(k), k=5,6$. As in Reference [15], $\left(\mathbf{H}_{3}\right)$ gives the bounds for $x^{\prime \prime \prime}(t)$. 
The auxiliary results which guarantee a priori bounds are given in Section 2, and the existence theorems are in Section 3. The ability to use $\left(\mathbf{H}_{\mathbf{1}}\right)$ and $\left(\mathbf{H}_{\mathbf{2}} \mathbf{)}\right.$ for studying the existence of solutions with important properties is shown in Appendix A. Examples are given in Section 4.

\section{Auxiliary Results}

This part ensures a priori bounds for the eventual $C^{3}[0,1]$-solutions of each family $(1)_{\lambda},(k), k=$ $\overline{2,6}$, that is, it ensures the constants $m_{i}, M_{i}, i=\overline{0,2}$, from (iv) of Lemma 1 and $\left(\mathbf{H}_{3}\right)$.

Lemma 2. Let $x \in C^{3}[a, b]$ be a solution to $(1)_{\lambda}$. Suppose $\left(\mathbf{H}_{\mathbf{1}}\right)$ holds with $[0,1]$ replaced by $[a, b]$ and $K=x^{\prime \prime}(b)$. Then

$$
F_{1}^{\prime} \leq x^{\prime \prime}(t) \leq L_{1}^{\prime} \text { on }[a, b]
$$

Proof. By contradiction, assume that $x^{\prime \prime}(t)>L_{1}^{\prime}$ for some $t \in[a, b)$. This means that the set

$$
S_{+}=\left\{t \in[a, b]: L_{1}^{\prime}<x^{\prime \prime}(t) \leq L_{2}^{\prime}\right\}
$$

is not empty because $x^{\prime \prime}(t)$ is continuous on $[a, b]$ and $x^{\prime \prime}(b) \leq L_{1}^{\prime}$. Besides, there is a $\gamma \in S_{+}$such that

$$
x^{\prime \prime \prime}(\gamma)<0
$$

As $x(t)$ is a $C^{3}[a, b]$ - solution to $(1)_{\lambda}$,

$$
x^{\prime \prime \prime}(\gamma)=\lambda f\left(\gamma, x(\gamma), x^{\prime}(\gamma), x^{\prime \prime}(\gamma)\right)
$$

But, $\left(\gamma, x(\gamma), x^{\prime}(\gamma), x^{\prime \prime}(\gamma)\right) \in S_{+} \times D_{x} \times D_{p} \times\left(L_{1}^{\prime}, L_{2}^{\prime}\right]$ and (9) imply

$$
x^{\prime \prime \prime}(\gamma) \geq 0,
$$

a contradiction. Consequently,

$$
x^{\prime \prime}(t) \leq L_{1}^{\prime} \text { for } t \in[a, b] .
$$

Along similar lines, assuming on the contrary that the set

$$
S_{-}=\left\{t \in[a, b]: F_{2}^{\prime} \leq x^{\prime \prime}(t)<F_{1}^{\prime}\right\}
$$

is not empty and using (10), we achieve a contradiction which implies that

$$
F_{1}^{\prime} \leq x^{\prime \prime}(t) \text { for } t \in[a, b] .
$$

The proof of the next assertion is virtually the same as that of Lemma 2 and is omitted; it can be found in [15].

Lemma 3. Let $x \in C^{3}[a, b]$ be a solution to $(1)_{\lambda}$. Suppose $\left(\mathbf{H}_{\mathbf{2}}\right)$ holds with $[0,1]$ replaced by $[a, b]$ and $K=x^{\prime \prime}(a)$. Then

$$
F_{1} \leq x^{\prime \prime}(t) \leq L_{1} \text { on }[a, b]
$$

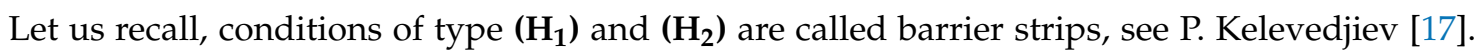
As can we see from Lemmas 2 and 3 they control the behavior of $x^{\prime \prime}(t)$ on $[a, b]$, depending on the sign of $f\left(t, x, x^{\prime}, x^{\prime \prime}\right)$ the curve of $x^{\prime \prime}(t)$ on $[a, b]$ crosses the strips $[a, b] \times\left[L_{1}^{\prime}, L_{2}^{\prime}\right],[a, b] \times\left[L_{1}, L_{2}\right],[a, b] \times\left[F_{2}^{\prime}, F_{1}^{\prime}\right]$ and $[a, b] \times\left[F_{2}, F_{1}\right]$ not more than once. This property ensures the a priori bounds for $x^{\prime \prime}(t)$. 
Lemma 4. Let $\left(\mathbf{H}_{\mathbf{1}}\right)$ hold for $K=C$. Then every solution $x \in C^{3}[0,1]$ to $(1)_{\lambda}$, (2) or (1) $\lambda$, (3) satisfies the bounds

$$
\begin{gathered}
|x(t)| \leq|A|+|B|+\max \left\{\left|F_{1}^{\prime}\right|,\left|L_{1}^{\prime}\right|\right\}, t \in[0,1] \\
\left|x^{\prime}(t)\right| \leq|B|+\max \left\{\left|F_{1}^{\prime}\right|,\left|L_{1}^{\prime}\right|\right\}, t \in[0,1] \\
F_{1}^{\prime} \leq x^{\prime \prime}(t) \leq L_{1}^{\prime}, t \in[0,1]
\end{gathered}
$$

Proof. Let first $x(t)$ be a solution to $(1)_{\lambda},(2)$. Using Lemma 2 we conclude that (11) is true. Then, according to the mean value theorem, for each $t \in[0,1)$ there is a $\xi \in(t, 1)$ such that

$$
x^{\prime}(1)-x^{\prime}(t)=x^{\prime \prime}(\xi)(1-t)
$$

which together with (11) gives the bound for $\left|x^{\prime}(t)\right|$. Again from the mean value theorem for each $t \in(0,1]$ there is an $\eta \in(0, t)$ with the property

$$
x(t)-x(0)=x^{\prime}(\eta) t
$$

which yields the bound for $|x(t)|$. The assertion follows similarly for $(1)_{\lambda},(3)$.

Lemma 5. Let $\left(\mathbf{H}_{\mathbf{1}}\right)$ hold for $K=C$. Then every solution $x \in C^{3}[0,1]$ to $(1)_{\lambda}$, (4) satisfies the bounds

$$
\begin{gathered}
|x(t)| \leq|A|+|B-A|+\max \left\{\left|F_{1}^{\prime}\right|,\left|L_{1}^{\prime}\right|\right\}, t \in[0,1], \\
\left|x^{\prime}(t)\right| \leq|B-A|+\max \left\{\left|F_{1}^{\prime}\right|,\left|L_{1}^{\prime}\right|\right\}, t \in[0,1], \\
F_{1}^{\prime} \leq x^{\prime \prime}(t) \leq L_{1}^{\prime}, t \in[0,1] .
\end{gathered}
$$

Proof. By Lemma $2, F_{1}^{\prime} \leq x^{\prime \prime}(t) \leq L_{1}^{\prime}$ on $[0,1]$. Clearly, there is a $\mu \in(0,1)$ for which $x^{\prime}(\mu)=B-A$. Further, for each $t \in[0, \mu)$ there is a $\xi \in(t, \mu)$ such that

$$
x^{\prime}(\mu)-x^{\prime}(t)=x^{\prime \prime}(\xi)(\mu-t)
$$

from where, using the obtained bounds for $x^{\prime \prime}(t)$, we get

$$
\left|x^{\prime}(t)\right| \leq|B-A|+\max \left\{\left|F_{1}^{\prime}\right|,\left|L_{1}^{\prime}\right|\right\}, t \in[0, \mu] .
$$

We can proceed analogously to see that the same bound is valid for $t \in[\mu, 1]$. Finally, for each $t \in(0,1]$ there is an $\eta \in(0, t)$ such that

$$
x(t)-x(0)=x^{\prime}(\eta) t
$$

which together with the obtained bound for $\left|x^{\prime}(t)\right|$ yields the bound for $|x(t)|$.

Lemma 6. Let $\left(\mathbf{H}_{\mathbf{1}}\right)$ and $\left(\mathbf{H}_{\mathbf{2}}\right)$ hold for $K=C-B$. Then every solution $x \in C^{3}[0,1]$ to $(1)_{\lambda},(5)$ or $(1)_{\lambda},(6)$ satisfies the bounds

$$
\begin{gathered}
|x(t)| \leq|A|+|B|+\max \left\{\left|F_{1}\right|,\left|L_{1}\right|,\left|F_{1}^{\prime}\right|,\left|L_{1}^{\prime}\right|\right\}, t \in[0,1], \\
\left|x^{\prime}(t)\right| \leq|B|+\max \left\{\left|F_{1}\right|,\left|L_{1}\right|,\left|F_{1}^{\prime}\right|,\left|L_{1}^{\prime}\right|\right\}, t \in[0,1], \\
\min \left\{F_{1}, F_{1}^{\prime}\right\} \leq x^{\prime \prime}(t) \leq \max \left\{L_{1}, L_{1}^{\prime}\right\}, t \in[0,1] .
\end{gathered}
$$


Proof. Let $x(t)$ be a solution to $(1)_{\lambda},(5)$; the proof is similar for $(1)_{\lambda},(6)$. We know there is a $v \in(0,1)$ for which $x^{\prime \prime}(v)=C-B$. Then, applying Lemmas 2 and 3 on the intervals $[0, v]$ and $[v, 1]$, respectively, we get

$$
F_{1}^{\prime} \leq x^{\prime \prime}(t) \leq L_{1}^{\prime} \text { on }[0, v] \text { and } F_{1} \leq x^{\prime \prime}(t) \leq L_{1} \text { on }[v, 1]
$$

and so the bounds for $x^{\prime \prime}(t)$ follow. Further, as in the proof of Lemma 4 we establish consecutively the bounds for $\left|x^{\prime}(t)\right|$ and $|x(t)|$.

\section{Existence Results}

Theorem 1. Let $\left(\mathbf{H}_{\mathbf{1}}\right)$ hold for $K=C$ and $\left(\mathbf{H}_{\mathbf{3}}\right)$ hold for

$$
\begin{gathered}
M_{0}=|A|+|B|+\max \left\{\left|F_{1}^{\prime}\right|,\left.\right|^{\prime} L_{1} \mid\right\}, m_{0}=-M_{0}, \\
M_{1}=|B|+\max \left\{\left|F_{1}^{\prime}\right|,\left|L_{1}^{\prime}\right|\right\}, m_{1}=-M_{1}, m_{2}=F_{1}^{\prime}, M_{2}=L_{1}^{\prime} .
\end{gathered}
$$

Then each of BVPs (1), (2) and (1), (3) has at least one solution in $C^{3}[0,1]$.

Proof. We will establish that the assertion is true for problem (1), (2) after checking that the hypotheses of Lemma 1 are fulfilled; it follows similarly and for (1), (3). We easily check that (i) holds for (1) , (2). Clearly, BVP (1), (2) is equivalent to BVP (1) $1,(2)$ and so (ii) is satisfied. Since now $\mathbf{L}_{h}=x^{\prime \prime \prime}$, (iii) also holds. Next, according to Lemma 4 , for each solution $x \in C^{3}[0,1]$ to $(1)_{\lambda}$, (2) we have

$$
m_{i} \leq x^{(i)}(t) \leq M_{i}, t \in[0,1], i=0,1,2 .
$$

Now use that $f$ is continuous on $[0,1] \times J$ to conclude that there are constants $m_{3}$ and $M_{3}$ such that

$$
m_{3} \leq \lambda f(t, x, p, q) \leq M_{3} \text { for } \lambda \in[0,1] \text { and }(t, x, p, q) \in[0,1] \times J,
$$

which together with $\left(x(t), x^{\prime}(t), x^{\prime \prime}(t)\right) \in J$ for $t \in[0,1]$ and Equation $(1)_{\lambda}$ implies

$$
m_{3} \leq x^{\prime \prime \prime}(t) \leq M_{3}, t \in[0,1]
$$

These observations imply that (iv) holds, too. Finally, the continuity of $f$ on the set $J$ gives $(v)$ and so the assertion is true by Lemma 1.

Theorem 2. Let $\left(\mathbf{H}_{\mathbf{1}}\right)$ hold for $K=C$ and $\left(\mathbf{H}_{3}\right)$ hold for

$$
\begin{gathered}
M_{0}=|A|+|B-A|+\max \left\{\left|F_{1}^{\prime}\right|,\left|L_{1}^{\prime}\right|\right\}, m_{0}=-M_{0}, \\
M_{1}=|B-A|+\max \left\{\left|F_{1}^{\prime}\right|,\left|L_{1}^{\prime}\right|\right\}, m_{1}=-M_{1}, m_{2}=F_{1}^{\prime}, M_{2}=L_{1}^{\prime} .
\end{gathered}
$$

Then BVP (1), (4) has at least one solution in $C^{3}[0,1]$.

Proof. It follows the lines of the proof of Theorem 1. Now the bounds

$$
m_{i} \leq x^{(i)}(t) \leq M_{i}, t \in[0,1], i=0,1,2,
$$

for each solution $x \in C^{3}[0,1]$ to a (1) $)_{\lambda}$ (4) follow from Lemma 5 .

Theorem 3. Let $\left(\mathbf{H}_{\mathbf{1}}\right)$ and $\left(\mathbf{H}_{\mathbf{2}}\right)$ hold for $\mathrm{K}=\mathrm{C}-\mathrm{B}$ and $\left(\mathbf{H}_{\mathbf{3}}\right)$ hold for

$$
\begin{gathered}
M_{0}=|A|+|B|+\max \left\{\left|F_{1}\right|,\left|L_{1}\right|,\left|F_{1}^{\prime}\right|,\left|L_{1}^{\prime}\right|\right\}, m_{0}=-M_{0}, \\
M_{1}=|B|+\max \left\{\left|F_{1}\right|,\left|L_{1}\right|,\left|F_{1}^{\prime}\right|,\left|L_{1}^{\prime}\right|\right\}, m_{1}=-M_{1},
\end{gathered}
$$




$$
m_{2}=\min \left\{F_{1}, F_{1}^{\prime}\right\}, M_{2}=\max \left\{L_{1}, L_{1}^{\prime}\right\} .
$$

Then each of BVPS (1), (5) and (1), (6) has at least one solution in $C^{3}[0,1]$.

Proof. Arguments similar to those in the proof of Theorem 1 yield the assertion. Now the bounds

$$
m_{i} \leq x^{(i)}(t) \leq M_{i}, t \in[0,1], i=0,1,2,
$$

for each solution $x \in C^{3}[0,1]$ to $(1)_{\lambda},(5)$ and (1) $\lambda_{\lambda},(6)$ follow from Lemma 6.

\section{Examples}

Through several examples we will illustrate the application of the obtained results.

Example 1. Consider the BVPs for the equation

$$
x^{\prime \prime \prime}(t)=\exp \left(x^{\prime \prime}-3\right)+5 x^{\prime \prime}\left(x^{\prime 2}+1\right)-t \sin x, t \in(0,1),
$$

with boundary conditions (2) or (3).

For $F_{2}^{\prime}=-|C|-2, F_{1}^{\prime}=-|C|-1, L_{1}^{\prime}=\max \{|C|, 3\}+1, L_{2}^{\prime}=\max \{|C|, 3\}+2$ and $\sigma=0.1$, for example, each of these problems has a solution in $C^{3}[0,1]$ by Theorem 1 .

Example 2. Consider the BVP

$$
\begin{gathered}
x^{\prime \prime \prime}(t)=\varphi\left(t, x, x^{\prime}\right)\left(\lg \left(\left(x^{\prime \prime}+50\right)\left(60-x^{\prime \prime}\right)\right)-3\right), t \in(0,1), \\
x(0)=5, x^{\prime}(0)=10, x^{\prime}(1)=40,
\end{gathered}
$$

where $\varphi:[0,1] \times \mathbb{R}^{2} \rightarrow \mathbb{R}$ is continuous and does not change its sign.

If $\varphi(t, x, p) \geq 0$ on $[0,1] \times \mathbb{R}^{2}$, the assumptions of Theorem 3 are satisfied for $F_{2}=-36, F_{1}=$ $-35, F_{2}^{\prime}=-46, F_{1}^{\prime}=-45, L_{1}^{\prime}=40, L_{2}^{\prime}=41, L_{1}=55, L_{2}=56$ and $\sigma=0.01$, for example, and if $\varphi(t, x, p) \leq 0$ on $[0,1] \times \mathbb{R}^{2}$, they are satisfied for $F_{2}^{\prime}=-36, F_{1}^{\prime}=-35, F_{2}=-46, F_{1}=-45$, $L_{1}=40, L_{2}=41, L_{1}^{\prime}=55, L_{2}^{\prime}=56$ and $\sigma=0.01$, for example; it is clear, $K=30$. Thus, the considered problem has at least one solution in $C^{3}[0,1]$. Let us note, here $D_{q}=(-50,60)$.

Example 3. Consider the BVP

$$
\begin{gathered}
x^{\prime \prime \prime}(t)=\frac{t\left(x^{\prime \prime}+8\right)\left(x^{\prime \prime}+3\right) \sqrt{625-x^{\prime 2}}}{\sqrt{900-x^{2}} \sqrt{100-x^{\prime \prime 2}}}, t \in(0,1), \\
x(0)=9, x(1)=1, x^{\prime \prime}(1)=-4 .
\end{gathered}
$$

For $F_{2}^{\prime}=-6, F_{1}^{\prime}=-5, L_{1}^{\prime}=-3, L_{2}^{\prime}=-2$ and $\sigma=0.1$, for example, this problem has a positive, decreasing, concave solution in $C^{3}[0,1]$ by Theorem A1; notice, here $D_{x}, D_{p}$ and $D_{q}$ are bounded.

Author Contributions: All authors contributed equally. All authors have read and agreed to the published version of the manuscript.

Funding: This research received no external funding.

Conflicts of Interest: The authors declare no conflict of interest. 


\section{Appendix A}

In this part we show how the barrier strips can be used for studying the existence of positive or non-negative, monotone, convex or concave $C^{3}[0,1]$ - solutions. Here, we demonstrate this on problem (1), (4) but it can be done for the rest of the BVPs considered in this paper. Similar results for various other two-point boundary conditions can be found in R. Agarwal and P. Kelevedjiev [16] and P. Kelevedjiev and T. Todorov [15].

Lemma A1. Let $A, B \geq 0, C \leq 0$. Suppose $\left(\mathbf{H}_{\mathbf{1}}\right)$ holds for $K=C$ with $L_{1}^{\prime} \leq 0$. Then each solution $x \in C^{3}[0,1]$ to $(1)_{\lambda},(4)$ satisfies the bounds

$$
\begin{gathered}
\min \{A, B\} \leq x(t) \leq A+|B-A|+\left|F_{1}^{\prime}\right|, t \in[0,1], \\
B-A+F_{1}^{\prime} \leq x^{\prime}(t) \leq B-A-F_{1}^{\prime}, t \in[0,1] .
\end{gathered}
$$

Proof. From Lemma 2 we know that $F_{1}^{\prime} \leq x^{\prime \prime}(t) \leq L_{1}^{\prime}$ for $t \in[0,1]$. Besides, for some $\mu \in(0,1)$ we have $x^{\prime}(\mu)=B-A$. Then,

$$
\int_{t}^{\mu} F_{1}^{\prime} d s \leq \int_{t}^{\mu} x^{\prime \prime}(s) d s \leq \int_{t}^{\mu} L_{1}^{\prime} d s, t \in[0, \mu),
$$

gives

$$
B-A \leq x^{\prime}(t) \leq B-A-F_{1}^{\prime}, t \in[0, \mu]
$$

and

$$
\int_{\mu}^{t} F_{1}^{\prime} d s \leq \int_{\mu}^{t} x^{\prime \prime}(s) d s \leq \int_{\mu}^{t} L_{1}^{\prime} d s, t \in(\mu, 1]
$$

implies

$$
B-A+F_{1}^{\prime} \leq x^{\prime}(t) \leq B-A, t \in[\mu, 1]
$$

As a result,

$$
B-A+F_{1}^{\prime} \leq x^{\prime}(t) \leq B-A-F_{1}^{\prime}, t \in[0,1] .
$$

Using Lemma 5, conclude

$$
|x(t)| \leq A+|B-A|+\left|F_{1}^{\prime}\right| \text { for } t \in[0,1] .
$$

From $x^{\prime \prime}(t) \leq L_{1}^{\prime} \leq 0$ for $t \in[0,1]$ it follows that $x(t)$ is concave on $[0,1]$ and so, in view of $A, B \geq 0, x(t) \geq \min \{A, B\}$ on $[0,1]$, which completes the proof.

Theorem A1. Let $A \geq B \geq 0$ and $C \leq 0(A \geq B>0$ and $C<0)$. Suppose $\left(\mathbf{H}_{\mathbf{1}}\right)$ holds for $K=C$ with $B-A \leq F_{1}^{\prime}\left(B-A<F_{1}^{\prime}\right)$ and $L_{1}^{\prime} \leq 0$, and $\left(\mathbf{H}_{3}\right)$ holds for

$$
\begin{gathered}
m_{0}=B, M_{0}=2 A-B+\left|F_{1}^{\prime}\right|, \\
m_{1}=B-A+F_{1}^{\prime}, M_{1}=B-A-F_{1}^{\prime}, m_{2}=F_{1}^{\prime}, M_{2}=L_{1}^{\prime} .
\end{gathered}
$$

Then BVP (1), (4) has at least one non-negative, non-increasing (positive, decreasing), concave solution in $C^{3}[0,1]$.

Proof. By Lemma 5, for every solution $x \in C^{3}[0,1]$ to $(1)_{\lambda}$, (4) we have $F_{1}^{\prime} \leq x^{\prime \prime}(t) \leq L_{1}^{\prime}$ on $[0,1]$, and Lemma A1 yields

$$
\begin{gathered}
B-A+F_{1}^{\prime} \leq x^{\prime}(t) \leq B-A-F_{1}^{\prime}, t \in[0,1] \\
\min \{A, B\} \leq x(t) \leq A+|B-A|+\left|F_{1}^{\prime}\right|, t \in[0,1] .
\end{gathered}
$$


Because of $A \geq B$, the last inequality gets the form

$$
B \leq x(t) \leq 2 A-B+\left|F_{1}^{\prime}\right|, t \in[0,1] .
$$

So, $x(t)$ satusfies the bounds

$$
m_{0} \leq x^{(i)}(t) \leq M_{0}, t \in[0,1], i=0,1,2 .
$$

Essentially the same reasoning as in the proof of Theorem 1 establishes that (1), (4) has a solution in $C^{3}[0,1]$. Since $m_{0}=B \geq 0\left(m_{0}>0\right), M_{1}=B-A-F_{1}^{\prime} \leq 0\left(M_{1}<0\right)$ and $M_{2}=L_{1}^{\prime} \leq 0$, this solution has the desired properties.

\section{Reference}

1. Li, H.; Feng, Y.; Bu, C. Non-conjugate boundary value problem of a third order differential equation. Electron. J. Qual. Theory Differ. Equ. 2015, 2015, 1-19. [CrossRef]

2. Li, S. Positive solutions of nonlinear singular third-order two-point boundary value problem. J. Math. Anal. Appl. 2006, 323, 413-425. [CrossRef]

3. Liu, Z.; Debnath, L.; Kang, S.M. Existence of monotone positive solutions to a third order two-point generalized right focal boundary value problem. Comput. Math. Appl. 2008, 55, 356-367. [CrossRef]

4. Liu, Z.; Ume, J.S.; Kang, S.M. Positive solutions of a singular nonlinear third order two-point boundary value problem. J. Math. Anal. Appl. 2007, 326, 589-601. [CrossRef]

5. Lin, X.; Zhao, Z. Sign-changing solution for a third-order boundary-value problem in ordered Banach space with lattice structure. Bound. Value Probl. 2014, 2014, 132. [CrossRef]

6. Smirnov, S. On the third order boundary value problems with asymmetric nonlinearity. Nonlinear Anal. Model. Control 2011, 16, 231-241. [CrossRef]

7. Yao, Q.; Feng, Y. The existence of solutions for a third-order two-point boundary value problem. Appl. Math. Lett. 2002, 15, 227-232. [CrossRef]

8. Feng, Y. Solution and positive solution of a semilinear third-order equation. J. Appl. Math. Comput. 2009, 29, 153-161. [CrossRef]

9. Feng, Y.; Liu, S. Solvability of a third-order two-point boundary value problem. Appl. Math. Lett. 2005, 18, 1034-1040. [CrossRef]

10. Feng, Y. Existence and uniqueness results for a third-order implicit differential equation. Comput. Math. Appl. 2008, 56, 2507-2514. [CrossRef]

11. Ma, R.; Lu, Y. Disconjugacy and extremal solutions of nonlinear third-order equations. Commun. Pure Appl. Anal. 2014, 13, 1223-1236. [CrossRef]

12. Granas, A.; Guenther, R.B.; Lee, J.W. Nonlinear Boundary Value Problems for Ordinary Differential Equations; Dissnes Math.: Warszawa, Poland, 1985.

13. Hopkins, B.; Kosmatov, N. Third-order boundary value problems with sign-changing solutions. Nonlinear Anal. 2007, 67, 126-137. [CrossRef]

14. Li, Y.; Li, Y. Positive solutions of a third-order boundary value problem with full nonlinearity. Mediterr. J. Math. 2017, 14, 128. [CrossRef]

15. Kelevedjiev, P.; Todorov, T.Z. Existence of solutions of nonlinear third-order two-point boundary value problems. Electron. J. Qual. Theory Differ. Equ. 2019, 2019, 1-15. [CrossRef]

16. Agarwal, R.P.; Kelevedjiev, P.S. On the solvability of fourth-order two-point boundary value problems. Mathematics 2020, 8, 603. [CrossRef]

17. Kelevedjiev, P. Existence of solutions for two-point boundary value problems Nonlinear Anal. 1994, 22, 217-224. [CrossRef] 